\title{
Quercitrin suppresses hepatocellular carcinoma metastasis and angiogenesis by targeting the Nrf2 signaling pathway
}

\author{
Hong Guang Li ${ }^{1}$, Heng Jun Gao ${ }^{1}$, Fang Feng Liu ${ }^{1}$ and Jun Liu ${ }^{1}$ \\ ${ }^{1}$ Department of Hepatobiliary Surgery, Shandong Provincial Hospital Affiliated to Shandong University, Jinan, Shandong, \\ China \\ Correspondence to: Jun Liu, email: liujunljun/@163.com
}

Keywords: HCC; quercitrin; metastasis; angiogenesis; Nrf2

Received: August 24, $2017 \quad$ Accepted: November 14, $2017 \quad$ Published: December 30, 2017

Copyright: Li et al. This is an open-access article distributed under the terms of the Creative Commons Attribution License 3.0 (CC BY 3.0), which permits unrestricted use, distribution, and reproduction in any medium, provided the original author and source are credited.

\section{ABSTRACT}

NF-E2-related factor 2 (Nrf2) abnormally accumulates in multi-types of cancer, and its expression is closely associated with a poor prognosis for cancer patients. A large body of research has demonstrated that Nrf2 can protect cancer cells from chemotherapeutic agents and facilitate the malignant progression of tumors. Quercitrin, which is found in flavonoids from Sedum sarmentosum, has been associated with potential anti-cancer roles in the initiation and progression of several types of cancer. In this current study, we mainly investigated whether quercitrin had a therapeutic effect against hepatocellular carcinoma (HCC) using in vitro and in vivo studies. Herein, we demonstrated that quercitrin inhibit human HCC cell growth, migration, and invasion. In vivo experiments showed the growth and metastasis of HCC cells was markedly inhibited by quercitrin treatment. Additionally, quercitrin inhibited HCC cell-induced mobility and invasion, as well as the tube formation of human umbilical vascular endothelial cells (HUVECs) in vitro. In vivo, quercitrin markedly suppressed microvessel density and increases HCC cell apoptosis in tumors from nude mice inoculated with HCC cells. Finally, we demonstrated that Nrf2 was involved in cell growth and metastasis in HCC cancer cells. In summary, we demonstrated for the first time that quercitrin inhibited human HCC growth, angiogenesis and metastasis by targeting the Nrf2 signaling pathway.

\section{INTRODUCTION}

Hepatocellular carcinoma (HCC) is one of the leading causes of cancer-associated deaths worldwide [1]. Although advances in HCC treatment, including hepatectomy and liver transplantation, have been achieved, the rapid development of HCC leads to a poor prognosis and a five-year survival rate below $5 \%$ [2]. The main challenges in treating HCC include early postoperative detection, distal metastasis, and intrahepatic recurrence. Cancer metastasis is a complex multi-step process that consists of a series of consecutive steps, including cell migration, invasion, and movement of cancer cells through circulation, as well as the reimplantation of cancer cells in distant organs, which can be followed by colonization and associated tumorinduced angiogenesis $[3,4]$. The ability to inhibit initial cancer cell migration and invasion will generally prevent metastasis. Therefore, improving our understanding of the molecular mechanisms of HCC metastasis may provide a new effective therapeutic target for the treatment of $\mathrm{HCC}$ and improve patient prognoses $[5,6]$.

NF-E2-related factor $2(\mathrm{Nrf2})$ is a critical regulator of various cytoprotective genes [7]. After translation, $\mathrm{Nrf2}$ is degraded by the proteolytic ubiquitin-proteasome system in the cytoplasm of cells. Kelch-like ECHassociated protein 1 (KEAP1) is a component of the Cullin 3 (CUL3)-based E3 ubiquitin ligase complex and it controls both Nrf2 stability and accumulation $[8,9]$. Abundant evidence indicates that $\mathrm{Nrf} 2$ either functions as a proto-oncogene or supports the transformation other oncogenes [10]. Additionally, Nrf2 promotes tumor cell survival under oxidative stress conditions and a potential model would posit that the up-regulation of Nrf2 activity 
could lead to tumor promotion by protecting cancer cells [11]. Moreover, the accumulation of Nrf2 in cancer cells provides an environment that facilitates cell growth and protects against chemotherapeutic agents and radiotherapy [12]. However, whether Nrf2 plays an important role in $\mathrm{HCC}$ cancer growth and metastasis has remained largely unknown to date.

The emergence of Chinese medicine provides a new option for the supplementation of synthetic anti-cancer drugs [13]. In various experimental models, quercitrin has been shown to exhibit anti-cancer activity, and is usually associated with the inhibition of cell proliferation and/or apoptosis induction [14]. In non-small cell lung cancer (NSCLC), previous studies have shown that quercetin can reduce NSCLC cell proliferation [15], induce G2/M phase arrest, and initiate apoptosis $[16,17]$. Other studies have demonstrated that quercetin has anti-proliferative and apoptotic effects on colon tissues and prostate cancer cells $[18,19]$. Although previous researches demonstrate that few flavonoids exhibit anti-cancer in $\mathrm{HCC}$, the anticancer roles of quercetin in HCC remain not well be elucidated [20]. Therefore, this present study aimed to determine whether quercetin could inhibit the growth and metastasis of HCC [21]. Additionally, we attempted to uncover the underlying cellular and molecular anticancer mechanisms of quercetin. Previous studies have shown that quercetin can inhibit the production of reactive oxygen species (ROS), enhance the activity of nuclear factor E2-related factor 2 (Nrf2), and drive antioxidant response element (ARE) activity in APAP-treated HepG2 cells. Consequently, we predicted that quercetin may inhibit HCC growth and metastasis by down-regulating the Nrf2 signaling pathway.

Herein, we demonstrated that quercetin inhibit HCC cell invasion and migration in vitro. Using the nude mouse model, we shown that quercetin inhibit the metastasis of HCC cells in vivo. To investigate how quercetin regulated cancer cells mechanistically, we evaluated the anti-cancer activity of quercetin on HCC cells and found that the inhibition of tumor growth and metastasis were related to the inhibition of the Nrf2 signaling pathway.

\section{RESULTS}

\section{Quercitrin inhibits HCC cell growth and induces apoptosis}

To assess the anti-cancer activity of quercetin, the HepG2 and PLC/PRF/5 HCC cell lines were used. As shown in Figure 1A, quercetin inhibited HepG2 and PLC/ $\mathrm{PRF} / 5$ cell proliferation in a dose- and time-dependent manner. The $\mathrm{IC}_{50}$ values for both HCC cells and incubation times were calculated, which showed that quercitrin exerted $50 \%$ inhibition under $20 \mu \mathrm{M}$ after 24 or $48 \mathrm{~h}$ treatment. We further confirmed the inhibitory effects on cell proliferation by colony formation analysis. After $24 \mathrm{~h}$ pre-treatment with quercetin, cells were incubated for three weeks and the colonies were counted. As shown in Figure $1 \mathrm{~B}$, quercetin markedly reduced the formation of $\mathrm{HCC}$ cell colonies in a concentration-dependent manner. To determine whether quercetin-mediated anti-cancer activity in HCC was associated with the induction of apoptosis, HepG2 and PLC/PRF/5 cells were treated with 2, 5, or $10 \mu \mathrm{M}$ quercetin for $24 \mathrm{~h}$. Apoptosis was then quantified by flow cytometry using Annexin V-FITC/PI double staining. As shown in Figure 1C, our data suggested that treatment with quercetin resulted in the dose-dependent induction of apoptosis in HepG2 and PLC/PRF/5 cells. In general, these data suggested that quercetin inhibit HCC cell growth and induces apoptosis.

\section{Quercitrin inhibits HCC cell invasion and migration}

To investigate the effect of quercetin on $\mathrm{HCC}$ metastasis in vitro, we determined the migration capacity of HepG2 and PLC/PRF/5 cells treated or not with quercetin. As shown in Figure 2A, the control group of cells closed the wounded area by $24 \mathrm{~h}$, whereas quercetin treatment significantly inhibited cell wound closure in a dose-dependent manner at $24 \mathrm{~h}$. Moreover, in Transwell invasion assays, quercetin treatment markedly reduced HepG2 and PLC/PRF/5 cell invasion (Figure 2B). Subsequent to this confirmation of the inhibition of quercitrin in $\mathrm{HCC}$ cell migration and invasion in vitro, we tested whether quercetin inhibited pulmonary metastasis by intravenously injecting $\mathrm{HCC}$ cells into nude mice by tail vein injection. In control mice, HCC cells metastasized to the lungs and formed multiple metastatic lesions, whereas in quercetin-treated mice the number of metastatic foci was significantly reduced (Figure 2C). These data suggested that quercetin effectively inhibited HCC cell metastasis in vivo. Epithelial interstitial transformation (EMT) is a key mechanism of cancer metastasis and is closely associated with cell migration and invasion. Thus, we analyzed the expression of the EMT-associated E-cadherin/N-cadherin by western blotting and qRT-PCR using the total cell lysates of HepG2 and PLC/PRF/5 cells that had been exposed to quercetin. Quercetin-treated HepG2 and PLC/PRF/5 cells showed significant downregulation of $\mathrm{N}$-cadherin and up-regulation of E-cadherin (Figure 2D). Together, these data suggested that quercetin treatment reduce the metastasis of $\mathrm{HCC}$ cells both in vitro and in vivo.

\section{Quercitrin suppresses HCC development in murine models}

To assess the anticancer effect of quercetin in vivo, we investigated the potential role of quercetin in a mouse model of HCC. Quercetin was orally administered, as shown in Figure 3A, and we found that 
quercetin significantly prolonged survival (Figure 3B) and inhibited tumor development (Figure 3C). Additionally, immunohistochemistry results suggested that Ki67 staining in cancer tissues could be reduced by the administration of quercetin (Figure 3D). Furthermore, quercetin treatment increased the percentage of caspase-3 positive cells in tumor tissues (Figure 3E), suggesting that quercetin induced tumor cell apoptosis. The CD31 index represents a marker of microvessel density. We finally tested whether quercetin could regulate tumor
A

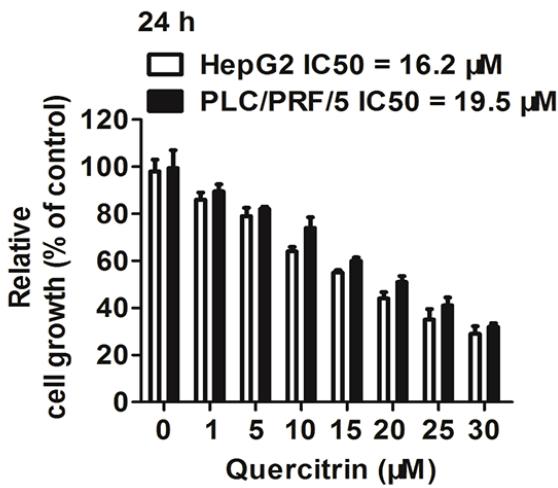

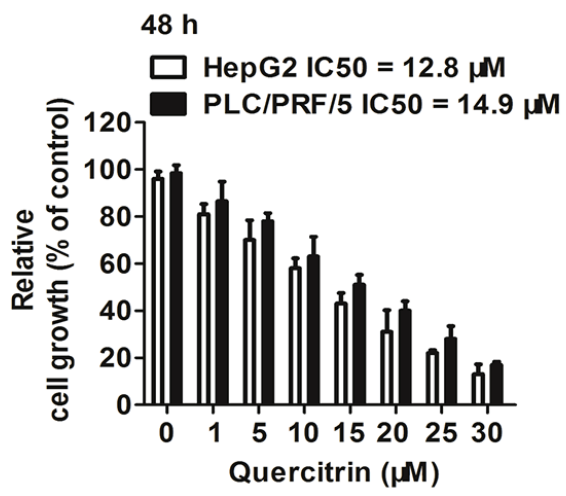

B
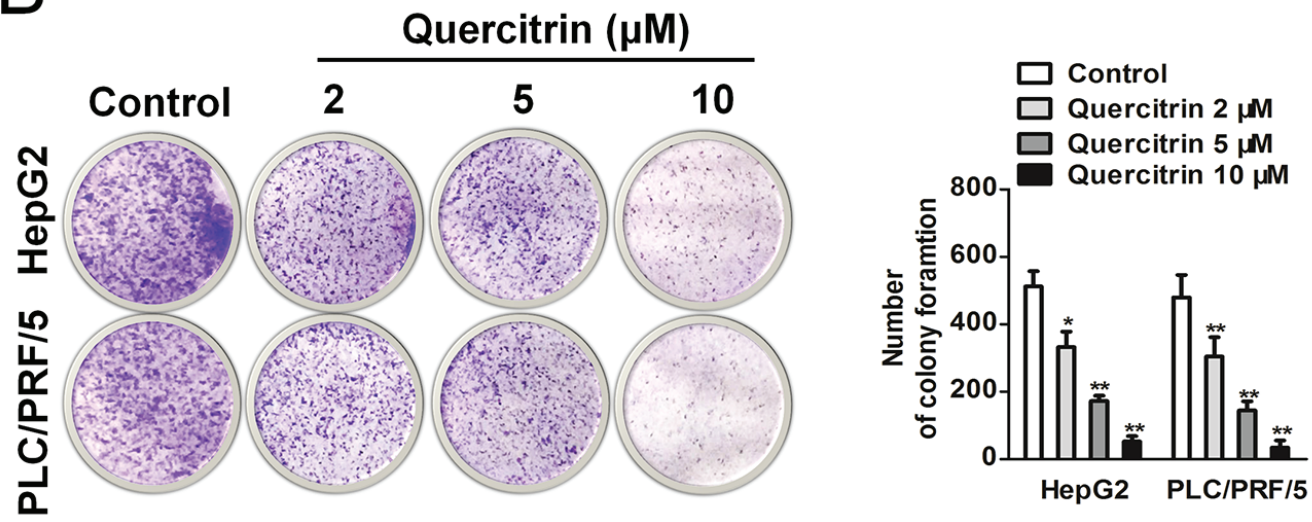

$\mathcal{C}$
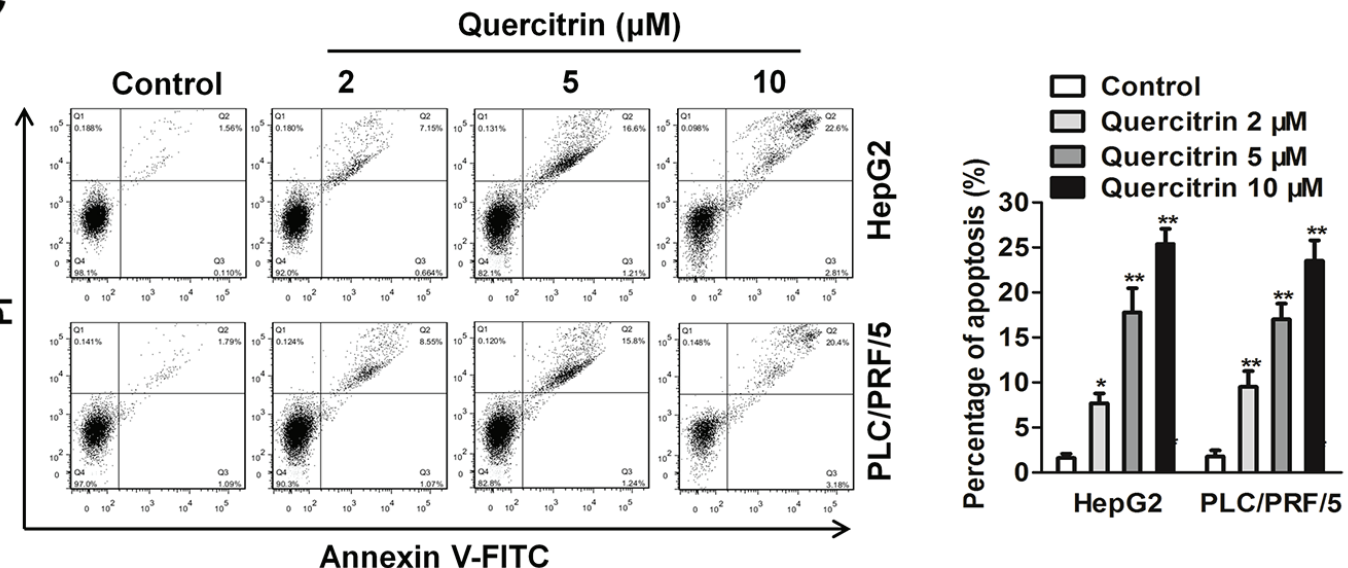

Figure 1: Quercitrin inhibits HCC cell proliferation. (A) HepG2 and PLC/PRF/5 cells were exposed to the indicated concentrations of quercitrin for 24 or $48 \mathrm{~h}$, and cell viability was determined. Data are presented as means $\pm \mathrm{SD}$. Values are expressed as the percentage of viable cells normalized to the percentage of viable cells in the untreated group. (B) Effects of quercitrin on HCC cell colony formation in vitro. HepG2 and PLC/PRF/5 cells were pre-treated with quercitrin for $24 \mathrm{~h}$. Cells were seeded in a 6 -well plate in the absence of quercitrin and were cultured for three weeks. (C) HepG2 and PLC/PRF/5 cells were treated with quercitrin and Annexin V-FITC/PI apoptosis assays were performed. Representative plots of apoptotic HepG2 and PLC/PRF/5 cells are shown. Data are presented as means $\pm \mathrm{SD} ;{ }^{*} p<0.05,{ }^{* *} p<0.01$ compared to untreated control cells. 
angiogenesis. Compared with the control group, we found that quercetin could down-regulate the expression of CD31 in HCC tumors (Figure 3F).

\section{Quercitrin suppressed tumor cell-induced angiogenesis}

Tumor-induced angiogenesis, which is critical to tumor cell growth, migration, invasion, and distant metastasis, depends upon angiogenic factors, including
VEGF-A and bFGF secreted by tumor cells. To determine the impact of quercetin on tumor angiogenesis, we found that human umbilical vein endothelial cells (HUVECs) formed tube-like structures on Matrigel after treatment with conditioned medium $(\mathrm{CM})$ from quercetin treated or untreated HepG2 or PLC/PRF/5 cells. As shown in Figure 4A, CM from both HepG2 and PLC/PRF/5 untreated quercetin cells induced obvious tube formation of HUVECs. However, quercetin-treated CM did not evoke a prominent increase in the tube network, suggesting that

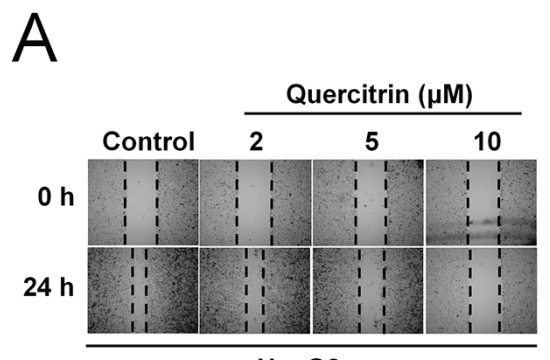

HepG2

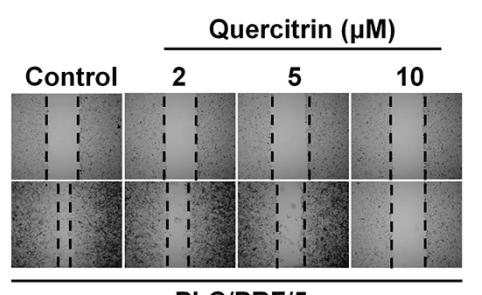

PLC/PRF/5

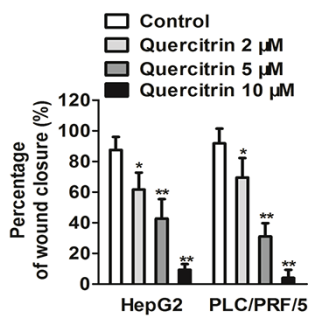

HepG2 PLC/PRF/5

B
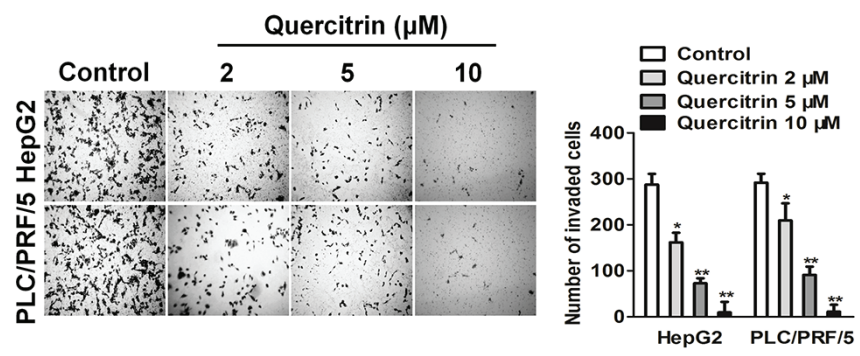

C
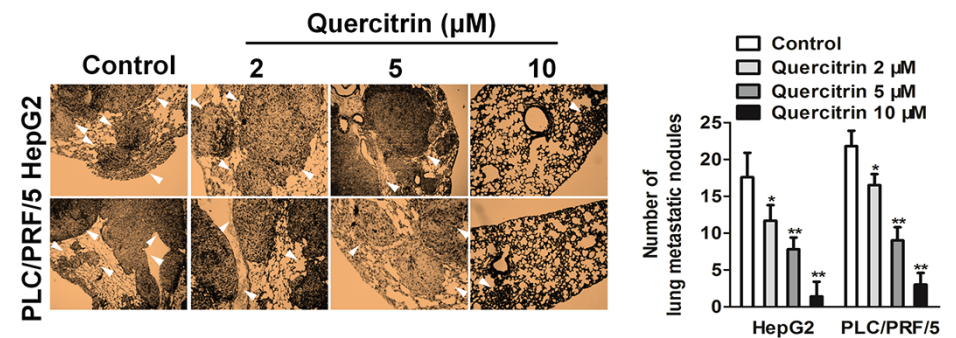

$D$
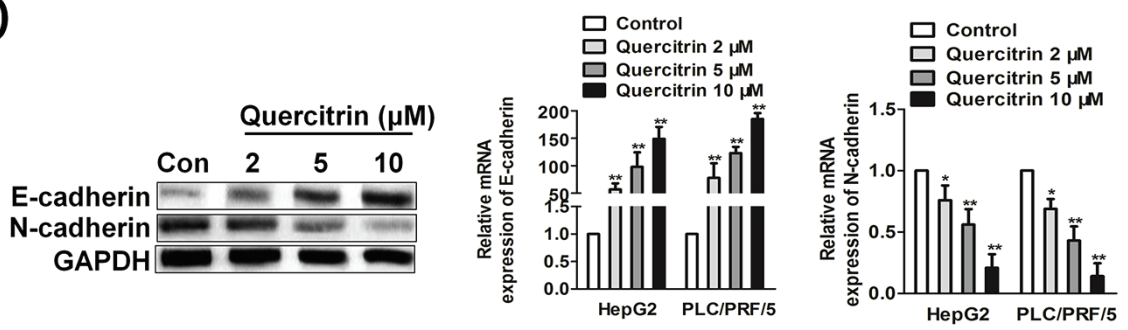

Figure 2: Quercitrin inhibits HCC cell metastasis. (A) HepG2 or PLC/PRF/5 cells were wounded by scraping with a pipette tip. Cells were incubated with quercitrin for $24 \mathrm{~h}$. The percentage of wound closure at $24 \mathrm{~h}$ was calculated based on the width of wound closure at $0 \mathrm{~h}$. (B) Cells pre-treated with quercitrin were allowed to invade the Transwell membrane. The cells that invaded were stained and quantified. Data are representative of three experiments and expressed as means $\pm \mathrm{SD}$ of five random fields per well. Data are presented as means $\pm \mathrm{SD} ;{ }^{*} p<0.05,{ }^{* *} p<0.01$ compared to untreated control. (C) BALB/c nude mice were injected with HepG2 or PLC/PRF/5 cells via the tail vein. After daily oral administration of vehicle (control) or quercitrin for 21 days, mice were sacrificed and lung tissues were subjected to H\&E staining. Images show metastatic colonies of the lungs. (D) Both HepG2 and PLC/PRF/5 cells were treated with quercitrin, and the expression levels of E-cadherin/N-cadherin were analyzed by western blotting and qRT-PCR. Data are presented as means $\pm \mathrm{SD} ;{ }^{*} p<0.05,{ }^{* *} p<0.01$ compared to untreated control cells. 
quercetin reduced the length of capillary-like structures in a dose-dependent manner. During the process of tumor angiogenesis, cancer cells vigorously attract endothelial cells by releasing angiogenic factors into microenvironment. To identify the effects of quercetin on tumor-mediated acceleration of endothelial cell mobility and invasion in vitro, HUVECs were inoculated in the upper chamber of the Transwell, whereas CM from quercetin-treated HepG2 or PLC/PRF/5 cells were plated in the lower chamber of the Transwell, respectively. As shown in Figure 4B, control CM in the lower chamber markedly induced HUVEC invasion. CM from quercetintreated HCC cells significantly inhibited HUVEC invasion, suggesting that quercetin inhibited tumor cellderived endothelial cell chemotaxis in a dose-dependent manner. As expected, the control group of cells that were treated with control CM closed the wounded area by $24 \mathrm{~h}$, whereas $\mathrm{CM}$ from quercetin-treated cells significantly delayed cell wound closure by $24 \mathrm{~h}$ (Figure 4C). Finally, we found that quercetin attenuated VEGF-A and bFGF expression, which regulated tumor angiogenesis. As shown in Figure 4D, quercetin significantly inhibited VEGF-A and bFGF levels in HCC cells in a dosedependent manner.

\section{Anti-cancer of quercitrin effects mediated by Nrf2 signaling}

We analyzed levels of Nrf2 in $47 \mathrm{HCC}$ cancer tissues and 29 cases of corresponding adjacent normal tissues by qRT-PCR. Nrf2 was more frequently found in HCC tissues than in adjacent normal tissues (Figure 5A). Western blot and qRT-PCR analysis also revealed overexpression of Nrf2 in HepG2, SNU-423, SNU-387, SNU-449 and PLC/ $\mathrm{PRF} / 5 \mathrm{HCC}$ cell lines in comparison to LO2 cell line (Figure 5B). To investigate whether Nrf2 promotes growth and metastasis in HCC cells, we knocked-down or overexpressed the Nrf2 gene in HepG2 or PLC/PRF/5 cells (data not shown). Our data demonstrated that the growth and invasion/migration of $\mathrm{HCC}$ cells could be significantly inhibited by Nrf2 knock-down (Figure 5C-5E), and increased by $\mathrm{Nrf} 2$ over-expression (Figure $5 \mathrm{~F}-5 \mathrm{H}$ ). To investigate whether quercitrin suppressed the growth and metastasis of HCC cells via Nrf2, a Nrf2 knock-down assay was conducted. Our findings suggested that the down-expression of Nrf2 markedly abolished quercitrininduced inhibition of $\mathrm{HCC}$ cell colony formation, migration, and invasion (Figure 6A-6C). Additionally, immunoblotting and qRT-PCR analysis demonstrated that

A

$B$
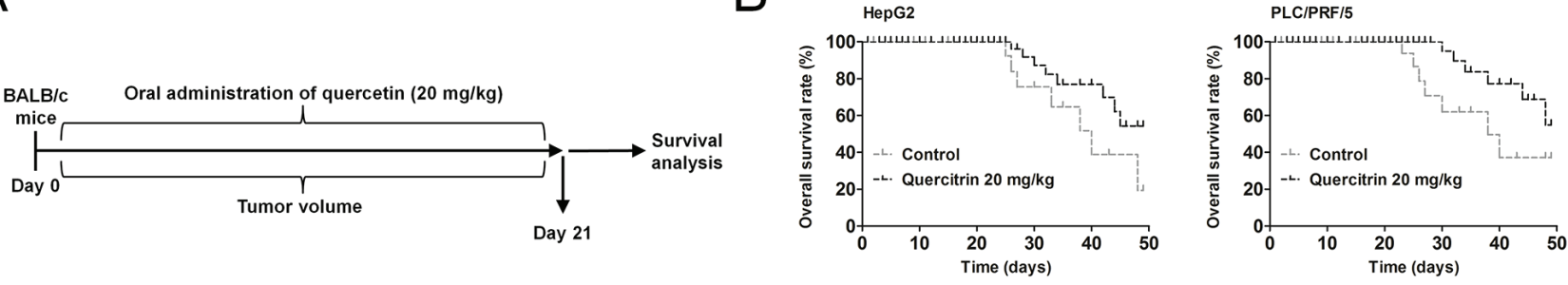

C

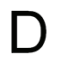

$E$

F
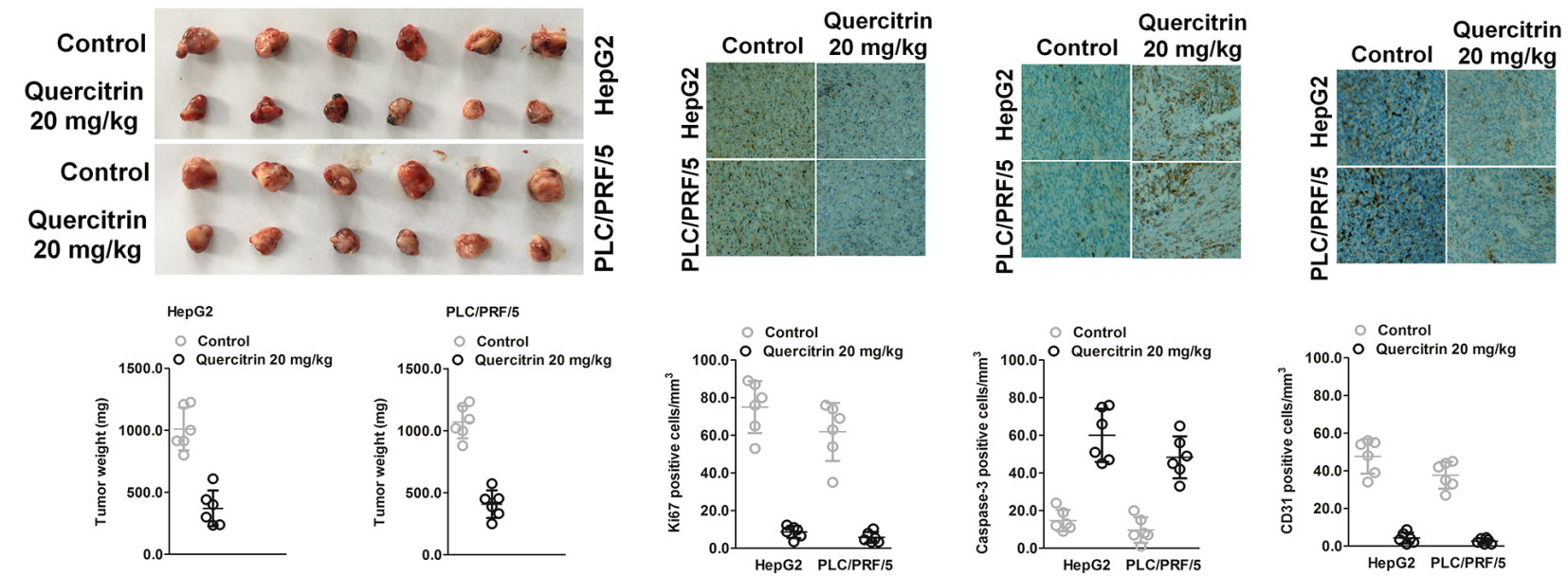

Figure 3: Quercitrin affects HCC cell growth in vivo. (A) As a murine HCC cancer model, mice were implanted with HCC cells subcutaneously and administered quercitrin, as shown in the schematic diagram. (B) The survival time was evaluated. (C) Tumor weight was evaluated. (D) Ki67 expression in tumor tissues. The percentage of positive cells is presented. Scale bar: $200 \mu \mathrm{m}$. (E) Caspase-3 expression in tumor tissues was evaluated by immunostaining. The percentage of positive cells was presented. (F) Immunohistochemical analysis of the tumor angiogenesis marker CD31 in tumor tissues. Percentages indicate positive staining for the indicated biomarker. 

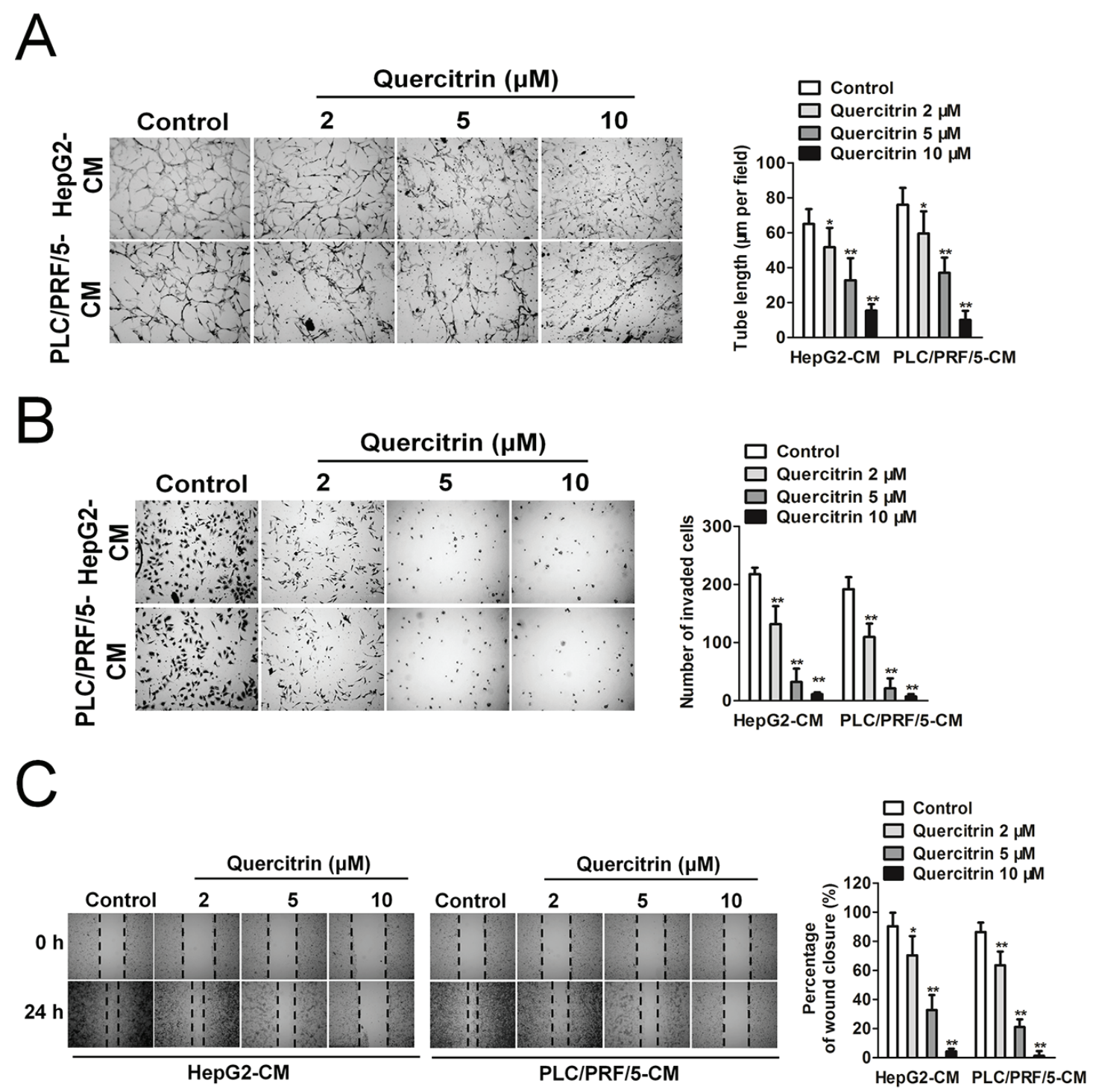

D
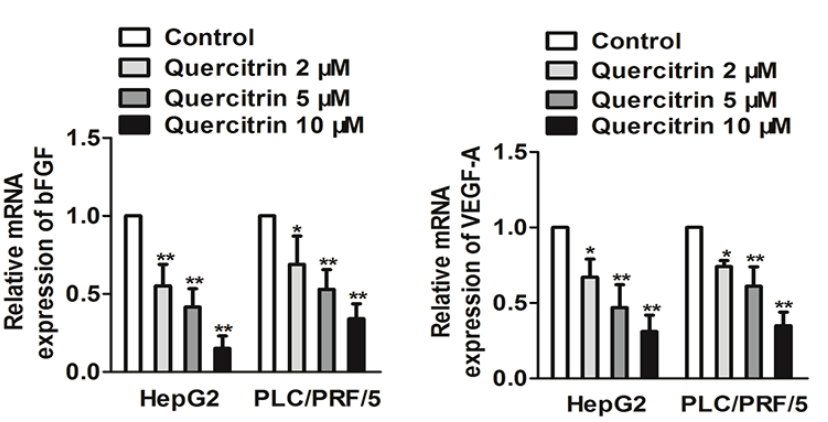

Figure 4: Quercitrin suppresses tumor cell-HUVECs tube formation, migration and invasion. (A) Tube formation by HUVECs induced by control CM or quercetin-treated HCC cells CM was examined. The total length of capillary-like structures was counted using ImageJ software. (B) CM from HepG2 or PLC/PRF/5 cells that were treated or not with quercitrin were plated into the lower chamber and HUVECs cells were added to the upper chamber. After $24 \mathrm{~h}$, HUVECs invaded were stained and quantified using ImageJ. (C) HUVECs cell monolayers were wounded by scraping. Cells were incubated for $24 \mathrm{~h}$ in the presence of control CM or quercetin-treated $\mathrm{CM}$ of HepG2 or PLC/PRF/5 cells. The percentage of wound closure at $24 \mathrm{~h}$ was calculated based on the width of the wound at $0 \mathrm{~h}$. Data are presented as means $\pm \mathrm{SD} ;{ }^{*} p<0.05,{ }^{* *} p<0.01$ compared to control CM. (D) HepG2 and PLC/PRF/5 cells were treated with quercitrin, and the expression levels of VEGF-A/bFGF were examined by western blotting and qRT-PCR. Data are presented as means \pm SD; ${ }^{*} p<0.05,{ }^{* *} p<0.01$ compared to controls. 
the down-expression of Nrf2 abrogated the influence of quercetin on E-cadherin/N-cadherin levels in HCC cells (Figure 6D). To confirm that quercitrin restrained tumorinduced angiogenesis was dependent on Nrf2, a Nrf2 knock-down experiment was conducted. We found that the effect of quercitrin on tumor-induced angiogenesis was abolished by Nrf2 knock-down (Figure 6E-6F). These data strongly implied that quercitrin suppressed the proliferation, invasion, and migration capacities of HCC cells by targeting Nrf2.

\section{A}

B

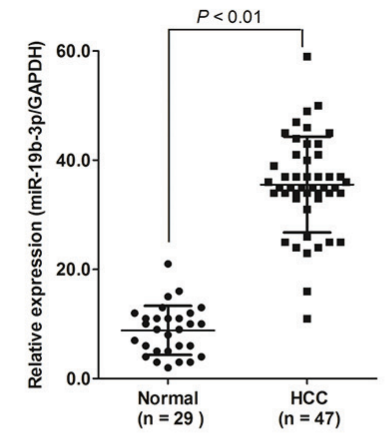

C
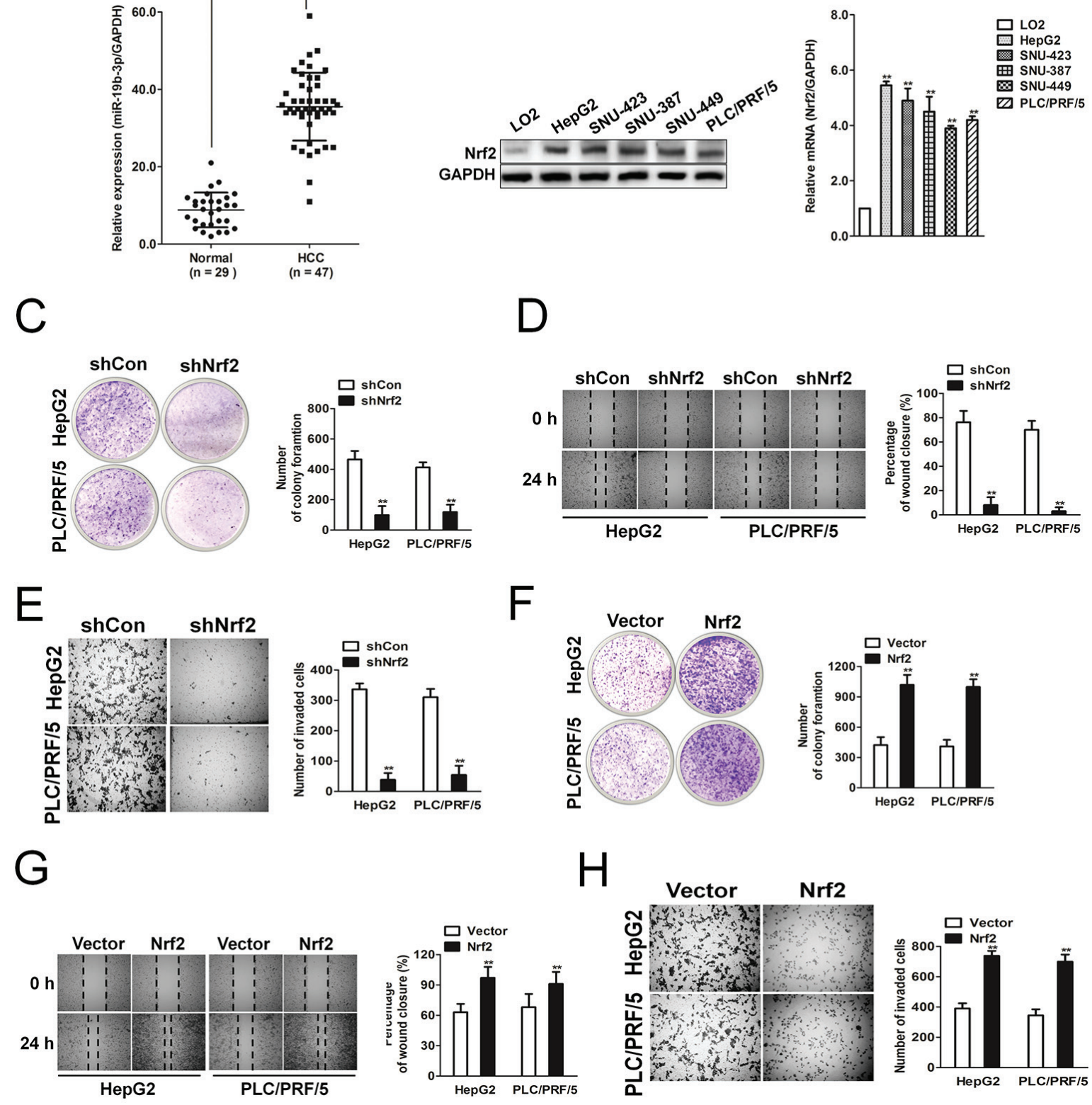

Figure 5: Effects of Nrf2 in the regulation of $\mathbf{H C C}$ cell growth and metastasis. (A) The mRNA expression levels of Nrf2 in HCC cancer tissues and corresponding adjacent normal tissues were determined by qRT-PCR. (B) Expression levels of Nrf2 in a panel of HCC cell lines compared to LO2 cells were assessed by immunoblotting and qRT-PCR. (C) Colony-formation assay. Colonies of HepG2 or PLC/PRF/5 cells transfected with shNrf2 were higher compared with those transfected with control shRNA. (D) HepG2 and PLC/ $\mathrm{PRF} / 5$ cells transfected with shNrf2 or control shRNA were wounded by scraping. The percentage of wound closure at $24 \mathrm{~h}$ was calculated on account of the width of the wound at 0 h. (E) HepG2 and PLC/PRF/5 cells that were transfected with shNrf2 or control shRNA were subjected to a Transwell invasion assay. Figures are representative of three experiments and are expressed as means \pm SD of five random fields per well. (F) Colony-formation analysis. Colonies of HepG2 or PLC/PRF/5 cells transfected with vector containing Nrf2 were lower compared with those transfected with the control vector. (G) HepG2 and PLC/PRF/5 cells were transfected with Nrf2 or control vector and were wounded by scraping. The percentage of wound closure at $24 \mathrm{~h}$ was calculated based on the width of the wound at $0 \mathrm{~h}$. (H) HepG2 and PLC/PRF/5 cells that were transfected with Nrf2 or vector were subjected to an invasion assay. 


\section{Overexpression of $\mathrm{Nrf} 2$ rescues $\mathrm{HCC}$ cell metastasis that could be suppressed by quercitrin}

To confirm that quercetin inhibited the migration and invasion of HCC cells by inhibiting Nrf2, Nrf2 rescue experiments were conducted. Our data suggested that the ectopic-expression of Nrf2 significantly attenuated quercetin-mediated inhibition of cell colony formation and metastasis by HepG2 and PLC/PRF/5 cells (Figure 7A-7C). Additionally, western blot analysis and qRT-PCR results revealed that the over-expression of Nrf2 reversed the effects of quercetin on E-cadherin/ $\mathrm{N}$-cadherin levels in HCC cells (Figure 7D). To confirm that quercetin inhibited tumor-induced angiogenesis, Nrf2, HepG2, or PLC/PRF/5 cells were transfected with a Nrf2 construct or an empty vector. In tube formation analysis, HUVECs formed tubelike structures on Matrigel, which could be rescued by the over-expression of Nrf2 in the presence of quercetin (Figure 7E). Finally, western blot and qRT-PCR analysis demonstrated that the over-expression of Nrf2 reversed the effects of quercitrin on VEGF-A and bFGF levels in HCC cells (Figure 7F). Our findings suggested that the overexpression of Nrf2 significantly reversed quercetinmediated angiogenesis inhibition.

\section{DISCUSSION}

Quercetin is an active ingredients in flavonoids that is abundantly found in Chinese herbs, onions, and fruits [22]. Extensive studies have demonstrated that quercitrin exhibits various biological activities, including antioxidant, anti-inflammatory, and anti-cancer properties [23]. Several studies suggest that the anti-tumor effects of quercitrin are mediated by inhibiting the metastatic potential of prostate cancers cells and melanoma and by restraining tumor angiogenesis $[24,25]$. Additionally, previous studies establish that quercitrin could suppress lipopolysaccharide- or 12-O-tetradecanoylphorbol13-acetate (TPA)-induced matrix metalloproteinase-9 (MMP-9) expression in glioma cells [26]. Although some pharmacological studies of quercitrin have been carried out [20], the anti-growth and anti-metastasis capacities of quercitrin in HCC have not yet well been analyzed. In this present study, we demonstrated that quercitrin could inhibit the migration, invasion, and metastasis of HCC cells in vitro and in vivo, and revealed the underlying molecular mechanisms. Quercitrin inhibited the expression of $\mathrm{Nrf2}$ and thereby suppressed $\mathrm{Nrf} 2$ target genes (E-cadherin/N-cadherin), leading to inhibition of the metastasis of HCC cells. Moreover, we confirmed
A
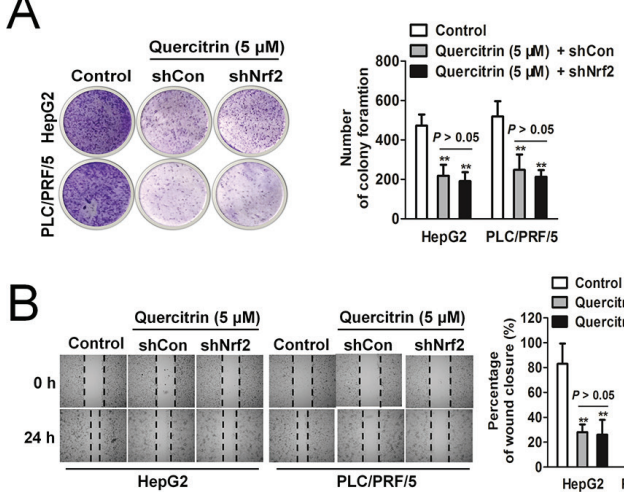
$\square$ Control
Quercitrin $(5 \mathrm{~m})+$ shCon
Q

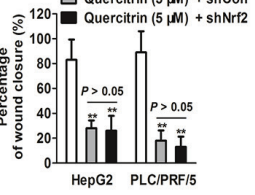

C
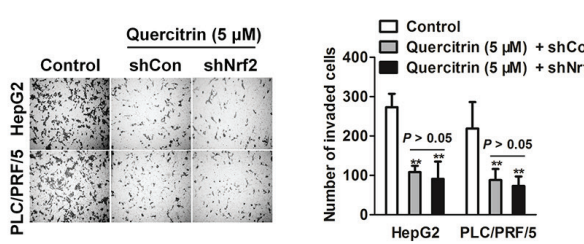

D
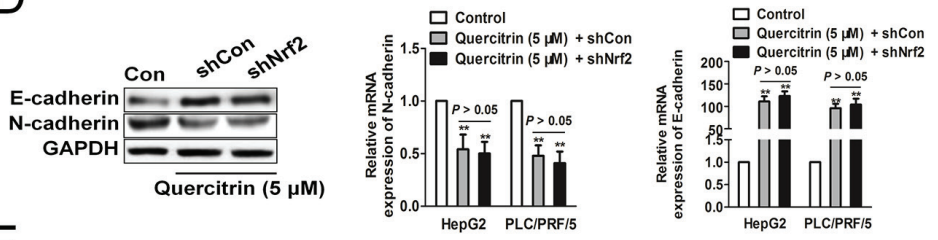

$E$

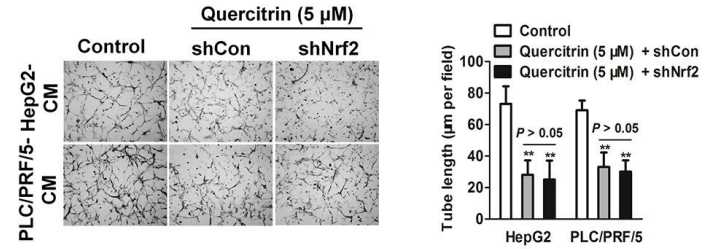

F
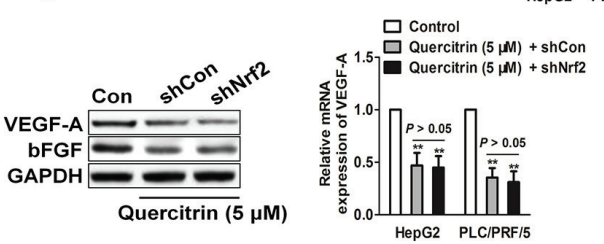

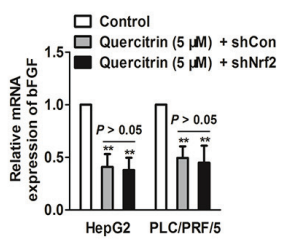

Figure 6: Quercitrin suppresses HCC cell metastasis and angiogenesis is dependent upon Nrf2. (A) Colony-formation assay. HepG2 or PLC/PRF/ 5 cells were transfected with shNrf2 or control shRNA and treated with $5 \mu \mathrm{M}$ quercitrin. (B) Wound healing assay. HepG2 or PLC/PRF/5 cells transfected with shNrf2 or control shRNA were treated with $5 \mu \mathrm{M}$ quercitrin. The percentage of wound closure at $24 \mathrm{~h}$ was calculated based on the width of the wound at $0 \mathrm{~h}$. (C) HepG2 or PLC/PRF/5 cells were subjected to Transwell invasion assays. Data are representative of three independent experiments and expressed as means \pm SD of five random fields per well. (D) The shNrf2 transfected HepG2 and PLC/PRF/5 cells were treated with quercitrin, and levels of E-cadherin/N-cadherin were examined by western blotting and qRT-PCR analysis. Data are presented as means $\pm \mathrm{SD} ;{ }^{* *} p<0.01$ compared to controls. (E) Tube formation of HUVECs. HepG2 or PLC/PRF/5 cells transfected with shNrf2 or control shRNA were treated with $5 \mu \mathrm{M}$ quercitrin. HUVECs were cultured with control $\mathrm{CM}$ or quercetin-treated $\mathrm{CM}$ of $\mathrm{HepG} 2$ or PLC/PRF/5 cells. The total length of capillary-like structures was measured using ImageJ. (F) The shNrf2 transfected HepG2 and PLC/PRF/5 cells were treated with quercitrin, and the expression levels of VEGF-A/ bFGF were examined by western blotting and qRT-PCR. Data are presented as means $\pm \mathrm{SD} ;{ }^{* *} p<0.01$ compared to controls. 
that quercitrin efficaciously suppressed $\mathrm{HCC}$ cell colony growth in vitro and xenograft tumor growth of HCC cells in vivo. Finally, we established an inhibitory effect of quercitrin on the angiogenic properties of HCC cells. Thus, our study suggested that quercitrin represents a novel candidate as an anti-cancer agent against HCC that was at least partially mediated by the inhibition of Nrf2 .

Cancer cell metastasis is a multi-step process, which includes local invasion, intravasation to blood systems, survival in the bloodstream, extravasation from the micro-vessels, and colonization at distant organs $[6,27]$. Many studies have yielded insights into the molecular mechanisms of each step of cancer cell metastasis [28]. The multistep process of metastasis and the known associated mechanisms provide numerous options for chemical interventions. Herein, we demonstrated that (1) quercitrin significantly attenuated HCC cell migration and invasion; (2) quercitrin suppressed HCC cell metastasis to the lung in vivo; and (3) quercitrin inhibited EMT-associated markers in HCC cells. Additionally, quercitrin markedly affected the prognosis and delayed tumor growth in a mouse xenograft model. Interestingly, quercitrin exhibited anti-angiogenic activities, whereas it exhibited lower CD31 expression in quercitrin-treated mouse. These data indicated that quercitrin may represent an angiogenesis inhibitor.
Solid tumors require persistent angiogenesis, which involves the formation of new vessels from pre-existing ones to allow for tumor cell growth and metastasis [29]. Without angiogenesis, cancer cell growth is restricted to a few millimeters and the cancer cells cannot escape into blood circulation to cause distant metastases [30]. In vivo, tumor-induced angiogenesis begins when tumor cells open an 'angiogenic switch' by releasing pro-angiogenic factors, including VEGF-A and bFGF, which, in turn, provoke pre-existing vessels to initiate angiogenesis [31]. In this present study, culture media (CM) from HCC cells were applied as an inducer of endothelial cells for an in vitro angiogenesis assay. In contrast to $\mathrm{CM}$ from $\mathrm{HCC}$ cells that were not treated with quercitrin, $\mathrm{CM}$ of quercitrin-treated HCC cells decreased the production of VEGF-A and bFGF by tumor cells; consequently, it inhibited the migration/invasion of endothelial cells. Quercitrin also suppressed the tumor-induced capillarylike tube formation of endothelial cells. Overall, these findings implied that quercitrin exhibited anti-angiogenic activities.

Furthermore, the expression of Nrf2 was assessed in $\mathrm{HCC}$ patient specimens and HCC cell lines. We found that its expression was elevated in HCC. The ectopic expression of Nrf2 promoted HCC cell metastasis, whereas silencing of Nrf2 resulted in inhibited metastasis.
A

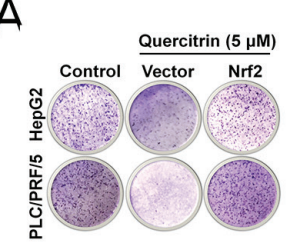

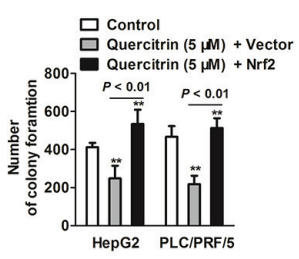

(1)

믄

B
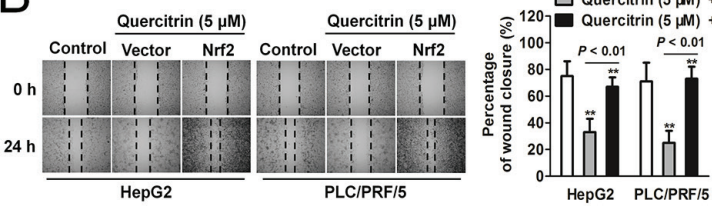

E
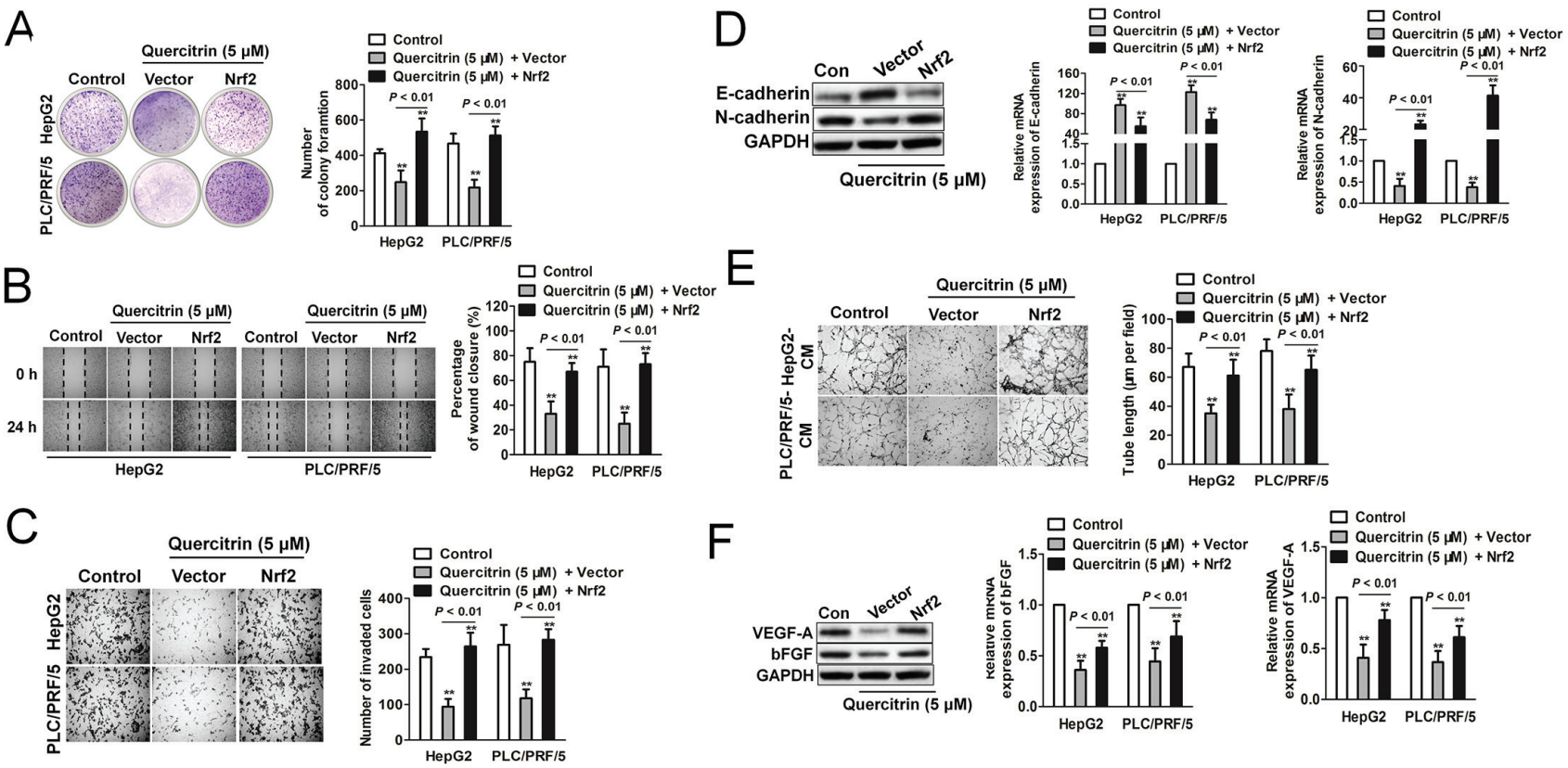

Figure 7: Upregulation of Nrf2 rescues the suppressive effects of quercetin on the growth and metastasis of HCC cells. (A) HepG2 and PLC/PRF/5 cells expressing Nrf2 or an empty vector were treated with $5 \mu \mathrm{M}$ quercitrin and a colony formation assay was performed. (B) Nrf2-overexpressing HCC cells were treated with or without $5 \mu \mathrm{M}$ quercitrin. Cellular migration was measured by wound healing assay. (C) HCC cells that overexpressed Nrf2 were treated with or without $5 \mu \mathrm{M}$ quercitrin. Cellular invasion was detected by a Transwell invasion assay. (D) Nrf2-overexpressing HepG2 and PLC/PRF/5 cells were treated with or without $5 \mu \mathrm{M}$ quercitrin. Expression levels of E-cadherin/N-cadherin were assessed by western blotting and qRT-PCR. (E) Tube formation by HUVECs induced by control CM or quercetin-treated CM from Nrf2-overexpressing HepG2 or PLC/PRF/5 cells were examined. The total length of capillary-like structures were measured using ImageJ. (F) HepG2 and PLC/PRF/5 cells that overexpressed Nrf2 or an empty vector were treated with or without 50 $\mu \mathrm{M}$ quercitrin. Expression levels of VEGF-A/bFGF were assessed by western blotting and qRT-PCR. Data are presented as means \pm SD; ${ }^{* *} p<0.01$ compared to control. 
Furthermore, the up-regulation of Nrf2 expression markedly stimulated altered expression of EMT-associated markers. Conversely, down-regulation of Nrf2 expression significantly decreased the expression of $\mathrm{N}$-cadherin and Nrf2 target genes. To determine whether quercitrin inhibited HCC metastasis and angiogenesis in a manner dependent on Nrf2, both Nrf2 knock-down and overexpression analyses were performed. We found that in the presence of Nrf2 shRNA, quercitrin did not influence HCC migration or invasion. We also found that quercitrin did not inhibit angiogenesis after HCC cells were transfected with Nrf2 shRNA. As expected, Nrf2 over-expression rescued the inhibitory effect of quercitrin on $\mathrm{HCC}$ metastasis and angiogenesis. Overall, we showed that quercitrin effectivity inhibited human HCC cell growth and metastasis. We also confirmed that quercitrin exerted inhibitory effects on the process of HCC cell-induced angiogenesis. As such, quercitrin exhibited the potential to be used as a potent anti-cancer agent in therapeutic strategies to inhibit the growth and metastasis of HCC.

\section{MATERIALS AND METHODS}

\section{Cell culture and HCC tissues}

HepG2, SNU-423, SNU-387, SNU-449 and PLC/ $\mathrm{PRF} / 5 \mathrm{HCC}$ cell lines and normal liver cells (LO2) were purchased from The Cell Bank of Type Culture Collection of Chinese Academy of Sciences (Shanghai, China). Cells were cultured in RMPI-1640 or DMEM supplemented with 10\% fetal bovine serum (FBS). In total, $47 \mathrm{HCC}$ tissues and 29 normal tissues were collected from Superchip Biotechnology Co. (Shanghai, China). The shRNA against Nrf2 (shNrf2, sc-37030-SH) and control shRNA (shCTL) were purchased from Santa Cruz Biotechnology (Dallas, TX, USA). HepG2 and PLC/ $\mathrm{PRF} / 5$ cells were seeded in 6-well plates the day prior to transfection and then were infected with shNrf2OR shCTL using Lipofectamine 2000 (Invitrogen). An Nrf2 expression plasmid (PCMV-Nrf2) was constructed with synthetic oligonucleotides and a PCMV vector (control vector) were purchased from Guangzhou FulenGen Co., Ltd (Guangzhou, Guangdong, China). HepG2 and PLC/ $\mathrm{PRF} / 5$ cells were transfected with Nrf2 plasmid or empty vector as control plasmids $(0.1 \mu \mathrm{g} /$ well for 96 well culture plates and $2 \mu \mathrm{g} /$ well for 6 well culture plates) using the X-tremeGENE HP DNA transfection reagent (Roche, Mannheim, Germany). After $48 \mathrm{~h}$ post-transfection, the cells were subjected to future experiments.

\section{Cell proliferation assay}

The MTT assay kit (Beyotime, Shanghai, China) was used to assess HCC cell viability after quercitrin treatment. Quercitrin was isolated from Sedum sarmentosum and purified in house; the purity exceeded $90.52 \%$, as determined by a HPLC method. Briefly, HepG2 or PLC/ $\mathrm{PRF} / 5$ cells were seeded into a 96-well plate overnight. Complete medium containing different concentrations of quercitrin was added into each well. Then, cells were cultured for 24 or $48 \mathrm{~h}$. Cell viability was measured by adding MTT agent and absorbance was determined using a Varioskan ${ }^{\mathrm{TM}}$ Flash Multimode Reader (Thermo Fisher Scientific, Waltham, MA, USA) at $490 \mathrm{~nm}$.

\section{Colony formation assay}

HepG2 or PLC/PRF/5 cells were pre-treated with quercitrin or DMSO for $24 \mathrm{~h}$ before seeding into a 6-well plate. After 21 days of culture, tumor colonies were visualized by $0.1 \%$ crystal violet staining and visible colonies were counted.

\section{Wound healing assay}

A confluent monolayer of HepG2, PLC/PRF/5, or HUVECs cells was wounded gently by scratching with a pipette tip. Complete media containing either quercitrin or DMSO was added into each well. For each group, two photographs were taken at 0 and $24 \mathrm{~h}$ after wound scratching. The percentage of wound closure in HCC cells at $24 \mathrm{~h}$ was calculated and compared to the width of the wound at $0 \mathrm{~h}$.

\section{Transwell assays}

Fresh media containing 15\% FBS was placed into the lower chamber of a Transwell (this acted as a chemoattractant). HepG2 or PL/PRF/5 cells were allowed to invade for $24 \mathrm{~h}$ with or without quercitrin. Then, invasive cells that had attached to the lower surface of the Transwell membrane were visualized by $0.1 \%$ crystal violet staining. Photographs of 5 random fields were selected, and the number of cells that invaded was counted.

\section{Apoptosis analysis}

Cell apoptosis was determined using an Annexin V-FITC apoptosis detection kit (KeyGen Biotechnology, Nanjing, China) according to the manufacturer's instructions. In brief, cells were treated with quercitrin for $24 \mathrm{~h}$. Cells were then harvested by trypsinization and washed twice with PBS. Cell pellets were re-suspended in binding buffer and stained with Annexin V-FITC/propidium iodide. After incubation at $15^{\circ} \mathrm{C}$ for $15 \mathrm{~min}$ in the dark, samples were analyzed using a Becton Dickinson FACSCalibur flow cytometer (Franklin Lakes, NJ, USA). Data were analyzed using CellQuest software (Becton Dickinson).

\section{qRT-PCR}

Total RNA of HCC cells or tissues was extracted using TRIZOL ${ }^{\circledR}$ reagent (Invitrogen), and $1 \mu \mathrm{g}$ RNA 
was used for cDNA synthesis with TaKaRa PrimeScript RT reagent kit (TaKaRa Biotechnology, Dalian, China). A Roche Light Cycler 480 PCR and SYBR ${ }^{\circledR}$ Premix Ex Taq $^{\mathrm{TM}}$ Green I (TaKaRa) were used for real-time PCR. The mRNA level of GAPDH was set as an internal control and the final mRNA levels of genes were normalized to those of the control group. Primer sequences of target genes were listed in Supplementary Table 1.

\section{Western blotting}

Protein samples were lysis using RIPA buffer. Proteins were separated by $10 \%$ SDS-PAGE and transferred to a PVDF membrane. After blocking with TBST containing 5\% non-fat milk powder, membranes were incubated overnight with anti-VEGF-A, anti-bFGF, anti-Nrf2, anti-E-cadherin, anti-N-cadherin (Cell Signaling Technology, MA, US), or anti-GAPDH (Cell Signaling Technology, MA, USA). Membranes were incubated with goat anti-rabbit secondary antibody (Boster, Wuhan, Hubei, China) for $2 \mathrm{~h}$ and enhanced chemiluminescence was (ECL) used to visualize protein bands with a Bio-Rad ChemiDoc XRS System.

\section{Xenograft model of HCC}

$\mathrm{BALB} / \mathrm{c}$ nude mice were purchased from the SLAKE Experimental Animal Company (SLAKE, Shanghai, China). The experimental protocol was approved by the Medical Experimental Animal Care Commission of Nanjing University of Chinese Medicine. A total of $6 \times 10^{6} \mathrm{HCC}$ cells were implanted by subcutaneous injection (100 $\mu \mathrm{l}$ per mouse) to yield subcutaneous tumors. Quercitrin $(20 \mathrm{mg} / \mathrm{kg})$ or vehicle ( $0.5 \%$ methylcellulose) was administered orally using a feeding tube. The tumor volume was calculated according to the following formula: Tumor volume $=$ (largest diameter $\times$ perpendicular height $\left.t^{2}\right) / 2$. Mice were euthanized at the end of the experiment to obtain tumor tissues. The tumor tissues were subjected to immunohistochemical staining with primary antibodies against Ki67, CD31, and caspase-3 (Abcam, Cambridge, MA, USA).

\section{Statistical analysis}

Data are presented as means \pm SD. Differences between two groups were evaluated using one-way ANOVA followed by Dunnett's post-hoc test. Differences with $P<0.05$ were considered to be statistically significant.

\section{ACKNOWLEDGMENTS AND FUNDING}

None.

\section{CONFLICTS OF INTEREST}

None.

\section{REFERENCES}

1. Kim JU, Cox IJ, Taylor-Robinson SD. The Quest for Relevant Hepatocellular Carcinoma Biomarkers. Cell Mol Gastroenterol Hepatol. 2017; 4:283-4.

2. Ogasawara S, Nakayama M, Akiba J, Kusano H, Yano H. Effect of sorafenib on des-gamma-carboxyprothrombin secretion by a human hepatocellular carcinoma cell line. Oncol Lett. 2017; 14:2170-6.

3. Sacco R, Tapete G, Simonetti N, Sellitri R, Natali V, Melissari S, Cabibbo G, Biscaglia L, Bresci G, Giacomelli L. Transarterial chemoembolization for the treatment of hepatocellular carcinoma: a review. J Hepatocell Carcinoma. 2017; 4:105-10.

4. Wang Z, Zou Q, Song M, Chen J. NEAT1 promotes cell proliferation and invasion in hepatocellular carcinoma by negative regulating miR-613 expression. Biomed Pharmacother. 2017; 94:612-8.

5. Shi W, Zhang Z, Yang B, Guo H, Jing L, Liu T, Luo Y, Liu H, Li Y, Gao Y. Overexpression of microRNA let-7 correlates with disease progression and poor prognosis in hepatocellular carcinoma. Medicine (Baltimore). 2017; 96:e7764.

6. Yuksel S, Boylu Akyerli C, Cengiz Yakicier M. Angiogenesis, Invasion, and Metastasis Characteristics of Hepatocellular Carcinoma. J Gastrointest Cancer. 2017 Aug 7. [Epub ahead of print].

7. Furfaro AL, Traverso N, Domenicotti C, Piras S, Moretta L, Marinari UM, Pronzato MA, Nitti M. The Nrf2/HO-1 Axis in Cancer Cell Growth and Chemoresistance. Oxid Med Cell Longev. 2016; 2016:1958174.

8. Leinonen HM, Kansanen E, Polonen P, Heinaniemi M, Levonen AL. Dysregulation of the Keap1-Nrf2 pathway in cancer. Biochem Soc Trans. 2015; 43:645-9.

9. Menegon S, Columbano A, Giordano S. The Dual Roles of NRF2 in Cancer. Trends Mol Med. 2016; 22:578-93.

10. Syu JP, Chi JT, Kung HN. Nrf2 is the key to chemotherapy resistance in MCF7 breast cancer cells under hypoxia. Oncotarget. 2016; 7:14659-72. https://doi.org/10.18632/ oncotarget.7406.

11. Tian Y, Liu Q, He X, Yuan X, Chen Y, Chu Q, Wu K. Emerging roles of Nrf2 signal in non-small cell lung cancer. J Hematol Oncol. 2016; 9:14.

12. Wang Y, Wang Y, Zhang Z, Park JY, Guo D, Liao H, Yi X, Zheng Y, Zhang D, Chambers SK, Zheng W. Mechanism of progestin resistance in endometrial precancer/cancer through Nrf2-AKR1C1 pathway. Oncotarget. 2016; 7:10363-72. https://doi.org/10.18632/oncotarget.7004.

13. Avila MA, Velasco JA, Cansado J, Notario V. Quercetin mediates the down-regulation of mutant p53 in the human breast cancer cell line MDA-MB468. Cancer Res. 1994; $54: 2424-8$.

14. Choi JA, Kim JY, Lee JY, Kang CM, Kwon HJ, Yoo YD, Kim TW, Lee YS, Lee SJ. Induction of cell cycle arrest and 
apoptosis in human breast cancer cells by quercetin. Int $\mathrm{J}$ Oncol. 2001; 19:837-44.

15. Xing N, Chen Y, Mitchell SH, Young CY. Quercetin inhibits the expression and function of the androgen receptor in LNCaP prostate cancer cells. Carcinogenesis. 2001; 22:409-14.

16. Elattar TM, Virji AS. The inhibitory effect of curcumin, genistein, quercetin and cisplatin on the growth of oral cancer cells in vitro. Anticancer Res. 2000; 20:1733-8.

17. Nakanoma T, Ueno M, Iida M, Hirata R, Deguchi N. Effects of quercetin on the heat-induced cytotoxicity of prostate cancer cells. Int J Urol. 2001; 8:623-30.

18. Ranelletti FO, Maggiano N, Serra FG, Ricci R, Larocca LM, Lanza P, Scambia G, Fattorossi A, Capelli A, Piantelli M. Quercetin inhibits p21-RAS expression in human colon cancer cell lines and in primary colorectal tumors. Int $\mathrm{J}$ Cancer. 2000; 85:438-45.

19. Scambia G, Panici PB, Ranelletti FO, Ferrandina G, De Vincenzo R, Piantelli M, Masciullo V, Bonanno G, Isola G, Mancuso S. Quercetin enhances transforming growth factor beta 1 secretion by human ovarian cancer cells. Int J Cancer. 1994; 57:211-5.

20. He CY, Fu J, Ma JY, Feng R, Tan XS, Huang M, Shou JW, Zhao ZX, Li XY, Zhang XF, Chen Y, Wang Y. Biotransformation and in vitro metabolic profile of bioactive extracts from a traditional Miao-nationality herbal medicine, Polygonum capitatum. Molecules. 2014; 19:10291-308.

21. Scambia G, Ranelletti FO, Panici PB, De Vincenzo R, Bonanno G, Ferrandina G, Piantelli M, Bussa S, Rumi C, Cianfriglia M, Mancuso S. Quercetin potentiates the effect of adriamycin in a multidrug-resistant $\mathrm{MCF}-7$ human breast-cancer cell line: P-glycoprotein as a possible target. Cancer Chemother Pharmacol. 1994; 34:459-64.

22. Yoshida M, Sakai T, Hosokawa N, Marui N, Matsumoto K, Fujioka A, Nishino H, Aoike A. The effect of quercetin on cell cycle progression and growth of human gastric cancer cells. FEBS Lett. 1990; 260:10-3.

23. Zheng SY, Li Y, Jiang D, Zhao J, Ge JF. Anticancer effect and apoptosis induction by quercetin in the human lung cancer cell line A-549. Mol Med Rep. 2012; 5:822-6.

24. Zhou J, Fang L, Liao J, Li L, Yao W, Xiong Z, Zhou X. Investigation of the anti-cancer effect of quercetin on HepG2 cells in vivo. PLoS One. 2017; 12:e172838.

25. Zhao X, Wang Q, Yang S, Chen C, Li X, Liu J, Zou Z, Cai D. Quercetin inhibits angiogenesis by targeting calcineurin in the xenograft model of human breast cancer. Eur $\mathrm{J}$ Pharmacol. 2016; 781:60-8.

26. Lou M, Zhang LN, Ji PG, Feng FQ, Liu JH, Yang C, Li BF, Wang L. Quercetin nanoparticles induced autophagy and apoptosis through AKT/ERK/Caspase-3 signaling pathway in human neuroglioma cells: In vitro and in vivo. Biomed Pharmacother. 2016; 84:1-9.

27. Pachmayr E, Treese C, Stein U. Underlying Mechanisms for Distant Metastasis - Molecular Biology. Visc Med. 2017; 33:11-20.

28. Lv G, Tan Y, Lv H, Fang T, Wang C, Li T, Yu Y, Hu C, Wen W, Wang H, Yang W. MXR7 facilitates liver cancer metastasis via epithelial-mesenchymal transition. Sci China Life Sci. 2017; 60:1203-1213.

29. Mashreghi M, Azarpara H, Bazaz MR, Jafari A, Masoudifar A, Mirzaei H, Jaafari MR. Angiogenesis biomarkers and their targeting ligands as potential targets for tumor angiogenesis. J Cell Physiol. 2017; 233:2949-2965.

30. Ronca R, Benkheil M, Mitola S, Struyf S, Liekens S. Tumor angiogenesis revisited: Regulators and clinical implications. Med Res Rev. 2017; 37:1231-1274.

31. Viallard C, Larrivee B. Tumor angiogenesis and vascular normalization: alternative therapeutic targets. Angiogenesis. 2017; 20:409-426. 